\title{
On Fuzzy gp*- neighborhood, interior and Closure in Fuzzy Topological Spaces
}

\section{Firdose Habib}

Department of mathematics Maulana Azad National Urdu University Hyderabad

\begin{tabular}{ll}
\hline ARTICLE INFO & ABSTRACT \\
\hline Published Online: & Based on fuzzy gp*-closed sets and fuzzy gp*-open sets, in this paper we have introduced fuzzy gp*- \\
06 March 2021 & neighborhoods, fuzzy gp*-Interior and fuzzy gp*-Closure. Also we investigated some of their \\
Corresponding Author: & elementary properties and discuss some important theorems of fuzzy gp*-neighborhoods, fuzzy gp*- \\
Firdose Habib & Interior and fuzzy gp*-Closure.
\end{tabular}

KEYWORDS: Fuzzy topological spaces; fuzzy gp*-closed sets; fuzzy gp*-open sets; fuzzy gp*- neighborhoods; fuzzy gp*Interior and fuzzy gp*-Closure.

\section{INTRODUCTION}

Introduced by Lotfi A. Zadeh in 1965[1] fuzzy set theory is the generalization of the classical set theory and thus fuzzy set extended the basic mathematical concept of a crisp set. So fuzzy mathematics is just a kind of mathematics developed in this framework and fuzzy topology is the generalization of topology in classical mathematics introduced by C.L Chang in 1968 [2]. Since then work started taking place in fuzzy topology at a rapid rate and various types of fuzzy sets were introduced and studied by various researchers, Like S.S Benchalli and G.P.Siddapur introduced fuzzy $\mathrm{g}^{*}$ pre continuous maps[4], Hamid Reza Moradi and Anahid Kamali introduced fuzzy strongly $\mathrm{g}^{*}$ closed sets and $\mathrm{g} * *$-closed sets in 2015 [5], In 2020 Firdose Habib and Khaja Moinuddin introduced fuzzy gp*-closed sets and fuzzy gp*-open sets [7]. And almost all the mathematical, engineering, medicinal etc. concepts have been redefined using fuzzy theory and it has further deepened the understanding of basic set theory.

Based on these newly introduced fuzzy gp*-closed and fuzzy gp*-open sets, in this paper we have introduced and studied fuzzy gp*- neighborhoods, fuzzy gp*-Interior and fuzzy gp*Closure. Also we investigated some of their elementary properties and discuss some important theorems.

\section{PRELIMINARIES}

Definition 2.1[1] Let $X$ be a space of objects, with a generic element of $X$ denoted by $x$. Then a fuzzy set $A$ in $X$ is a set of ordered pairs $\left\{\left(x, f_{A}(x)\right)\right\}$ where $f_{A}(x)$ is called the membership function which associates each point in $X$ a real number in the interval $[0,1]$.

Definition 2.2 [2] A family $\tau$ of fuzzy sets of $X$ is called fuzzy topology on $X$ if 0 and 1 belong to $\tau$ and $\tau$ is closed with respect to arbitrary union and finite intersection. The elements of $\tau$ are called fuzzy open sets and there complements are called fuzzy closed sets. The space $X$ with topology $\tau$ is called fuzzy topological space denoted by ( $X$ $, \tau)$.

Definition 2.3 [2] For a fuzzy set $\alpha$ of $\mathrm{X}$, the closure $\mathrm{Cl} \alpha$ and the interior Int $\alpha$ of $\alpha$ are defined respectively, as $C l \alpha$ $=\wedge\{\mu: \mu \geq \alpha, 1-\mu \in \tau\}$ and Int $\alpha=\mathrm{V}\{\mu: \mu \leq \alpha, \mu \in \tau\}$

Definition 2.4 [2] A function $f$ from a fts $(X, \tau)$ to a fts $(Y, \delta)$ is fuzzy-continuous iff the inverse of each $\delta$-open fuzzy set in $Y$ is $\tau$-open fuzzy set in $X$.

Definition 2.5 [2] let $(X, \tau)$ be a fts. A fuzzy set $h$ in $X$ is a neighborhood of fuzzy set $\alpha$ in $X$ iff there is $g \in \tau$ such that $\alpha \leq g \leq h$.

Definition 2.6 [3] A fuzzy set $n$ in a fts $(X, \tau)$ is a neighborhood of a point $x \in X$ iff there is $g \in \tau$ such that $g \leq n$ and $\mathrm{n}(\mathrm{x})=\mathrm{g}(\mathrm{x})>0$. A neighborhood of a point $x$ is frequently denoted by $n_{x}$. A neighborhood $n_{x}$ is called an open neighborhood of $x$ iff $n_{x} \in \tau$.

Definition 2.7 [2] Let $A$ and $B$ be fuzzy sets in $\mathrm{fts}(X, \tau)$, and let $A \geq B$. Then $B$ is called an interior fuzzy set of $A$ iff $A$ is 
nbhd of $B$. The union of all interior fuzzy sets of $A$ is called the interior of $A$ and is denoted by $A$.

Definition 2.8 [2] Let $A$ and $B$ be two fuzzy sets in a space $X$ $=\{\mathrm{x}\}$ with the grades of membership of $\mathrm{x}$ in $A$ and $B$ denoted by $\mu_{A}(\mathrm{x})$ and $\mu_{B}(\mathrm{x})$, respectively. Then

$\mathrm{A}=\mathrm{B} \Leftrightarrow \mu_{A}(\mathrm{x})=\mu_{B}(\mathrm{x})$ for all $\mathrm{x} \varepsilon \mathrm{X}$

$\mathrm{A} \subset \mathrm{B} \Leftrightarrow \mu_{A}(\mathrm{x}) \leq \mu_{B}(\mathrm{x})$ for all $\mathrm{x} \varepsilon \mathrm{X}$

$\mathrm{C}=\mathrm{A} \quad \mathrm{B} \Leftrightarrow \mu_{C}(\mathrm{x})=\operatorname{Max}\left[\mu_{A}(\mathrm{x}), \mu_{B}(\mathrm{x})\right]$ for all $\mathrm{x} \varepsilon \mathrm{X}$

$\mathrm{D}=\mathrm{A} \quad \mathrm{B} \Leftrightarrow \mu_{D}(\mathrm{x})=\operatorname{Min}\left[\mu_{A}(\mathrm{x}), \mu_{B}(\mathrm{x})\right]$ for all $\mathrm{x} \varepsilon \mathrm{X}$

Definition 2.9 [6] A fuzzy set A of a fuzzy topological space $(\mathrm{X}, \tau)$ is called fuzzy generalized pre-closed or gp-closed set if $\operatorname{pcl}(A) \leq U$ whenever $A \leq U$ and $\mathrm{U}$ is a fuzzy open set in $(X, \tau$ ). And complement of a Fuzzy gp-closed set is called fuzzy generalized pre-open or gp-open set.

Definition 2.10 [7] A fuzzy set $\lambda$ of a fuzzy topological space $(\mathrm{Y}, \tau)$ is called fuzzy generalized pre star closed (briefly fuzzy gp*-closed) if $\operatorname{cl}(\lambda) \leq \mu$

whenever $\lambda \leq \mu$ and $\mu$ is fuzzy generalized pre-open in $Y$.

Definition 2.11 [7] suppose a fuzzy set $\lambda$ is fuzzy generalized pre star closed set in fuzzy topological space $(Y, \tau)$, Then its complement I.e. $1-\lambda$ is called fuzzy generalized pre star open ( briefly fuzzy gp*-open) in $(Y, \tau)$.

Remark 2.12 [7] All fuzzy closed sets are fuzzy gp* closed sets.

Remark 2.13 [7] All fuzzy open sets are fuzzy gp*-open.

\section{FUZZY GENERALIZED PRE STAR (FUZZY gp*) NEIGHBORHOOD}

Definition 4.1 Suppose $(Y, \tau)$ is a fuzzy topological space \& suppose that $y \in Y$. Then a subset $N$ of $Y$ is called fuzzy $g p^{*}$ neighborhood of $y$ if and only if there exists a fuzzy gp*-open subset $M$ of $Y$ such that $y \in M \leq N$.

Definition 4.2 Suppose $(Y, \tau)$ is a fuzzy topological space then a subset $N$ of $Y$ is said to be fuzzy gp *-neighborhood of a fuzzy set $L$ in $Y$ if and only if there exists a fuzzy gp*-open set $M$ in $Y$ such that $L \leq M \leq N$.

Theorem 4.3 Every fuzzy neighborhood $\alpha$ of $y \in Y$ is fuzzy $g{ }^{*}$-neighborhood of $y$.

Proof: Suppose $\alpha$ is a fuzzy neighborhood of point $y \in Y$. So by definition of fuzzy neighborhood, we have a fuzzy open set $\mu$ in $Y$ such that $y \in \mu \leq \alpha$. Now as every fuzzy open set is fuzzy gp*-open in Y, implies $\mu$ is fuzzy gp*-open set. So $y \in$ $\mu \leq \alpha$, where $\mu$ is fuzzy gp*-open set implies $\alpha$ is fuzzy gp*neighborhood of $y$.

Theorem $4.4 \alpha$ is fuzzy gp*-neighborhood of each of its points in fuzzy topological space $(Y, \tau)$, if $\alpha$ is fuzzy gp*-open in $Y$.
Proof: Let $\alpha$ is a fuzzy gp*-open set in $Y$ and let $y \in \alpha$. Now, the proof that $\alpha$ is fuzzy gp*-neighborhood of $y$ is clear as $y$ $\in \alpha \leq \alpha$, and $\alpha$ is fuzzy gp*-open set in $Y$. So $\alpha$ follows the definition of fuzzy $\mathrm{gp}^{*}$ - neighborhood. Now as $y$ is any arbitrary point in $\alpha$ implies that $\alpha$ is fuzzy gp*-neighborhood of each of its points.

Theorem 4.5 Suppose $(Y, \tau)$ is a fuzzy topological space \& A is a fuzzy gp*-closed subset of $Y$, and let $y \in 1-A$. Then $\exists$ fuzzy gp*-neighborhood $B$ of y such that $A \wedge B=\phi$.

Proof: Given that $A$ is a fuzzy gp*-closed subset of $Y$, implies $1-A$ is a fuzzy gp*-open subset in $Y$, such that $y \in 1-$ $A$. So by Theorem $4.4,(1-A)$ is a fuzzy gp*-neighborhood of $y$. Now by Definition $4.2 \exists$ a fuzzy gp*-open set $B$, which is also fuzzy gp*-neighborhood of $y$ (by Theorem 4.4) in $Y$ such that $y \in B \leq 1-A$ I.e. $A \wedge B=\phi$.

\section{FUZZY GENERALIZED PRE STAR INTERIOR (FUZZY gp*-INT).}

Definition 5.1 Suppose $(Y, \tau)$ is a fuzzy topological space and $\alpha$ is a fuzzy subset in $Y$. Then a point $y \in \alpha$ is called fuzzy $g p^{*}$ interior point of $\alpha$, if $\alpha$ is fuzzy gp*-nbhd of $y$. The collection of all fuzzy gp*- interior points of $\alpha$ is said to be fuzzy $g p^{*}$ interior of $\alpha$, denoted by fuzzy $g p^{*}$-int $(\alpha)$.

Theorem 5.2 Suppose $(Y, \tau)$ is a fuzzy topological space and $\alpha, \mu$ be two fuzzy subsets of $Y$. Then we have

(a) Fuzzy $g p^{*}-\operatorname{int}\left(1_{Y}\right)=1_{Y} \quad \&$ fuzzy $g p^{*}$-int $\left(0_{Y}\right)=0_{Y}$.

(b) Fuzzy $g p^{*-\operatorname{int}}(\alpha) \leq \alpha$.

(c) Suppose $\alpha$ is any fuzzy gp*-open set contained in $\mu$, Then $\alpha \leq$ fuzzygp ${ }^{*}$-int $(\mu)$

(d) Suppose $\alpha \leq \mu$, Then fuzzy gp ${ }^{*}$-int $(\alpha) \leq$ fuzzy gp ${ }^{*}$-int $(\mu)$

Proof: (a) We know that $1_{Y}$ and $0_{Y}$ are fuzzy gp*-open sets in $Y$, as every fuzzy open set is fuzzy gp*- open. So fuzzy gp*-int $\left(1_{Y}\right)=\vee\left\{\mathrm{H}: \mathrm{H}\right.$ is fuzzy gp*-open $\left.\mathrm{H} \leq 1_{Y}\right\}$

$\Rightarrow$ Fuzzy gp*-int $\left(1_{Y}\right)=1_{Y} \vee\{$ all fuzzy gp*-open sets $\}$

$\Rightarrow$ Fuzzy gp*-int $\left(1_{Y}\right)=1_{Y}$.

Now, as $0_{Y}$ is the only fuzzy gp*-open set contained in ${ }^{0} Y$. So fuzzy gp*-int $\left(0_{Y}\right)=0_{Y}$

(b) Suppose y $\in$ fuzzy gp*-int $(\alpha)$

$$
\begin{aligned}
& \Rightarrow \mathrm{y} \text { is fuzzy interior point of } \alpha \text {. } \\
& \Rightarrow \alpha \text { is fuzzy neighborhood of } y \text {. } \\
& \Rightarrow \mathrm{y} \in \alpha .
\end{aligned}
$$

So, $y \in$ fuzzy gp*-int $(\alpha) \Rightarrow y \in \alpha$, I.e. fuzzy gp*-int $(\alpha) \leq \alpha$.

(c) Suppose that $\alpha$ is any fuzzy gp*-open set contained in $\mu$. Now, let $y \in \alpha$. So as $\alpha$ is fuzzy gp*-open set contained in $\mu$, implies $y$ is fuzzy gp*-interior point of $\mu$. I.e. $y \in g p^{*}$-int $(\mu)$. Hence $\alpha \leq g p^{*}$-int $(\mu)$.

(d) Suppose $\alpha \& \mu$ are two fuzzy subsets of fuzzy 
topological space $(y, \tau)$, such that $\alpha \leq \mu$. Now we have to show that

$$
\text { fuzzy } g p^{*}-\operatorname{int}(\alpha) \leq \text { fuzzy } g p^{*} \text {-int }(\mu) .
$$

Let $y \in$ fuzzy gp*-int $(\alpha)$, which implies $y$ is fuzzy gp*interior point of $\alpha$ I.e. $\alpha$ is fuzzy gp*-nbhd of $y$. Now as $\alpha \leq \mu$, implies $\mu$ is also fuzzy gp*-nbhd of y I.e. $y \in$ fuzzy gp*-int $(\mu)$.

So $y \in$ fuzzy gp*-int $(\alpha) \Rightarrow y \in$ fuzzy gp*-int $(\mu)$ I.e.

$$
\text { fuzzy } g p^{*}-\operatorname{int}(\alpha) \leq \text { fuzzy } g p^{*} \text {-int }(\mu) \text {. }
$$

Theorem 5.3 Suppose $(Y, \tau)$ is a fuzzy topological space \& $\alpha$ is any fuzzy $g p^{*}$-open subset in $Y$, then fuzzy $g p^{*}$-int $(\alpha)=\alpha$.

Proof: Suppose $\alpha$ is a fuzzy gp*-open subset in $Y$. Now from Theorem 5.2(b) we have fuzzy $g p^{*}-\operatorname{int}(\alpha) \leq \alpha$. Also $\alpha$ is fuzzy gp*-open subset contained in $\alpha$. So by Theorem 5.2 (c), we have $\alpha \leq$ fuzzy $g p^{*}$-int $(\alpha)$. Hence fuzzy $g p^{*}$-int $(\alpha)=\alpha$.

Theorem 5.4 Suppose $(Y, \tau)$ is a fuzzy topological space \& $\alpha$, $\mu$ are fuzzy subsets of $Y$. Then, fuzzy $g p^{*}$-int $(\alpha) \vee$ fuzzy $g p^{*}$ $\operatorname{int}(\mu) \leq$ fuzzy $g p^{*}$-int $(\alpha \vee \mu)$.

Proof: We have $\alpha \leq \alpha \vee \mu \& \mu \leq \alpha \vee \mu$. Now by Theorem 5.2(d) we have,

$$
\begin{gathered}
\text { Fuzzy } g p^{*} \text {-int }(\alpha) \leq \text { fuzzy } g p^{*} \text {-int }(\alpha \vee \mu) \& \\
\text { Fuzzy } g p^{*} \text {-int }(\mu) \leq \text { fuzzy } g p^{*} \text {-int }(\alpha \vee \mu)
\end{gathered}
$$

The above two inequalities implies that,

$$
\text { Fuzzy } g p^{*} \text {-int }(\alpha) \vee f u z z y g p^{*} \text {-int }(\mu) \leq f u z z y g p^{*} \text {-int }(\alpha \vee \mu) \text {. }
$$

Theorem 5.5 Suppose $(Y, \tau)$ is a fuzzy topological space $\& \alpha$, $\mu$ are fuzzy subsets of $Y$. Then fuzzy $g p^{*}$-int $(\alpha \wedge \mu)=$ fuzzy $g p^{*-i n t}(\alpha) \wedge$ fuzzy $g p^{*-i n t}(\mu)$.

Proof: Since we know that $\alpha \wedge \mu \leq \alpha$ and $\alpha \wedge \mu \leq \mu$, so by Theorem 5.2(d) we have

$$
\begin{gathered}
\text { Fuzzy } g p^{*-i n t}(\alpha \wedge \mu) \leq f u z z y g p^{*} \text {-int }(\alpha) \quad \& \\
\text { Fuzzy } g p^{*-i n t}(\alpha \wedge \mu) \leq f u z z y g p^{*} \text {-int }(\mu)
\end{gathered}
$$

The above two inequalities imply that

$$
\begin{gathered}
\text { Fuzzy } g p^{*} \text {-int }(\alpha \wedge \mu) \leq f u z z y g p^{*} \text { int }(\alpha) \wedge f u z z y g p^{*} \text {-int } \\
(\mu) \rightarrow(1)
\end{gathered}
$$

Now, Let $y \in$ fuzzy $g p^{*}$-int $(\alpha) \wedge$ fuzzy $g p^{*}$-int $(\mu)$.

$$
\Rightarrow \quad \mathrm{y} \in \text { fuzzy } g p^{*} \text {-int }(\alpha) \& y \in f u z z y g p^{*} \text {-int }(\mu) .
$$

So it follows that $y$ is fuzzy gp*-interior point of both the sets $\alpha \& \mu$. Which implies that both $\alpha \& \mu$ are fuzzy gp*nbhds of $y$. Implying that $\alpha \wedge \mu$ is also fuzzy gp*neighborhood of $y$.

$$
\Rightarrow y \in \text { fuzzy gp*-int }(\alpha \wedge \mu) \text {. Therefore, }
$$

Fuzzy $g p^{*-i n t}(\alpha) \wedge$ fuzzy $g p^{*-i n t}(\mu) \leq$ fuzzy gp*-int $(\alpha \wedge \mu)$

$$
\rightarrow(2)
$$

From (1) and (2), we have

Fuzzy $g p *_{\text {-int }}(\alpha \wedge \mu)=$ fuzzy $g p^{*}$-int $(\alpha) \wedge$ fuzzy $g p^{*}$-int $(\mu)$.

Theorem 5.6 Let $(Y, \tau)$ be a fuzzy topological space and $\alpha$ is a fuzzy subset of $Y$, Then fuzzy $g p^{*}$-int $(\alpha)=\vee\{\mu: \mu$ is a fuzzy gp*-open set in $Y$ and $\mu \leq \alpha\}$.

Proof: Suppose that $\alpha$ is a fuzzy subset of $Y$. Now we know that,

$y \in$ fuzzy gp*-int $(\alpha) \Leftrightarrow y$ is a fuzzy gp*-interior point of $\alpha$. $\Leftrightarrow \alpha$ is fuzzy gp*-nbhd of $y$. $\Leftrightarrow \exists$ a fuzzy gp*-open set $\mu$ such that $y$ $\in \mu \leq \alpha$ (by Definition 4.1)

$\Leftrightarrow \mathrm{y} \in \vee\{\mu: \mu$ is a fuzzy gp*-open set in Y such that $y \in \mu \leq \alpha$.

$\Rightarrow$ fuzzy gp*-int $(\alpha)=\vee \vee\{\mu: \mu$ is a fuzzy gp*-open set in $Y$ and $\mu \leq \alpha\}$.

Theorem 5.7 Suppose $(Y, \tau)$ is a fuzzy topological space $\& \alpha$ is any fuzzy subset in $Y$. Then, fuzzy int $(\alpha) \leq f u z z y g p^{*}-\operatorname{int}(\alpha)$.

Proof: Suppose that $\alpha$ is any fuzzy subset of $Y$. And let $y \in$ fuzzy $\operatorname{int}(\alpha)$.

$$
\begin{array}{ll}
\Rightarrow & y \in \vee\{\mu: \mu \text { is fuzzy open in } Y, \mu \leq \alpha\} \\
\Rightarrow & \exists \text { A fuzzy open set } \mu \text { such that } y \in \mu \leq \alpha
\end{array}
$$

Now as every fuzzy open set is fuzzy gp*-open, Implies there exists a fuzzy gp*-open set $\mu$ such that $y \in \mu \leq \alpha$.

$$
\begin{aligned}
& \Rightarrow \quad y \in \vee\{\mu: \mu \text { is fuzzy gp*-open in } Y, \mu \leq \alpha\} \\
& \Rightarrow \quad y \in \text { fuzzy } \operatorname{gp}^{*}-\operatorname{int}(\alpha) .
\end{aligned}
$$

So $y \in$ fuzzy int $(\alpha) \Rightarrow y \in$ fuzzy gp*-int $(\alpha)$ I.e. fuzzy int $(\alpha) \leq$ fuzzy $g p^{*}$-int $(\alpha)$

\section{FUZZY GENERALIZED PRE STAR CLOSURE (FUZZY gp*-CLOSURE).}

Definition 6.1 Suppose $(Y, \tau)$ is a fuzzy topological space and $\alpha \leq Y$. Then fuzzy $g p^{*}$-closure of $\alpha$ is defined as fuzzy $g p^{*}$-cl $(\alpha)=\wedge\left\{\mu: \alpha \leq \mu, \mu\right.$ is fuzzy gp ${ }^{*}$-closed set in $\left.Y\right\}$

Theorem 6.2 Suppose $(Y, \tau)$ is a fuzzy topological space and $\alpha, \mu$ are fuzzy subsets of $Y$. Then

(a) fuzzy $g p^{*_{-}} \operatorname{cl}\left(1_{Y}\right)=1_{Y}$ and fuzzy $g p^{*_{-}} \operatorname{cl}\left(0_{Y}\right)=0_{Y}$.

(b) $\alpha \leq$ fuzzy $g p^{*}-\operatorname{cl}(\alpha)$.

(c) Suppose $\mu \leq \alpha$, where $\alpha$ is fuzzy gp*-closed set. Then fuzzy $\mathrm{gp}^{*}-\operatorname{cl}(\mu) \leq \alpha$.

(d) If $\alpha \leq \mu$, then fuzzy $g p^{*}-\operatorname{cl}(\alpha) \leq$ fuzzy $g p^{*}-\operatorname{cl}(\mu)$. 
Proof: (a) We know that fuzzy $\mathrm{gp}^{*}-\mathrm{cl}\left(1_{Y}\right)$ is the intersection I.e. minimum of all fuzzy gp*-closed sets in $Y$ containing ${ }^{1} Y$ and since $1_{Y}$ is the minimum fuzzy gp*-closed set containing $1_{Y}$. So fuzzy $g p^{*}-\operatorname{cl}\left(1_{Y}\right)=1_{Y}$. Now fuzzy $\mathrm{gp}^{*}-\mathrm{cl}\left(0_{Y}\right)$ is the intersection I.e. minimum of all fuzzy $\mathrm{gp}^{*}$-closed sets in $\mathrm{Y}$ containing $0_{Y}$ and we know that $0_{Y}$ is the minimum fuzzy gp*-closed set containing $0_{Y}$. So fuzzy $g p^{*}$ - $c l\left(0_{Y}\right)=0_{Y}$.

Proof: (b) Since we know that fuzzy $\mathrm{gp}^{*}-\operatorname{cl}(\alpha)$ is the intersection of all fuzzy gp*-closed sets containing $\alpha$. So $\alpha \leq$ fuzzy $g p^{*}-\operatorname{cl}(\alpha)$ is obvious

Proof: (c) Suppose $\mu \leq \alpha$, where $\alpha$ is fuzzy gp*-closed set. Now,

\section{fuzzy $g p^{*}-c l(\mu)=\wedge\{\pi: \mu \leq \pi, \pi$ is fuzzy gp*-closed set in $Y\}$}

I.e. fuzzy $\mathrm{gp}^{*}$-cl $(\mu)$ is contained in all fuzzy gp*-closed sets, so in particular fuzzy $\mathrm{gp}^{*}-\mathrm{cl}(\mu) \leq \alpha$

Proof: (d) Suppose $\alpha \leq \mu$, and we know that fuzzy $g p^{*}-c l(\mu)=\wedge\{\pi: \mu \leq \pi, \pi$ is fuzzy gp*-closed set in $Y\}$ $\rightarrow(d .1)$

Now if $\mu \leq \pi$, where $\pi$ is fuzzy gp*-closed in $Y$, then by (c) of this theorem we have fuzzy $g p^{*}-c l(\mu) \leq \pi$. Now by (b) of this theorem $\mu \leq$ fuzzy $g p^{*}$-cl $(\mu)$ implies $\alpha \leq \mu \leq \pi$, where $\pi$ is fuzzy gp*-closed. So we have fuzzy $g p^{*}-\operatorname{cl}(\alpha) \leq \pi$ (by (c) of this theorem). Therefore

fuzzy $g p^{*}$-cl $(\alpha) \leq \wedge\{\pi: \mu \leq \pi, \pi$ is fuzzy gp*-closed set in $Y\}$

$\Rightarrow \quad$ fuzzy $g p^{*}$-cl $(\alpha) \leq f u z z y g p^{*}-c l(\mu) . \quad(u \operatorname{sing}(d .1))$

Theorem 6.3 Suppose $(Y, \tau)$ is a fuzzy topological space and $\alpha$ is a fuzzy $g p^{*}$-closed set in $Y$, then fuzzy $g p^{*}-\operatorname{cl}(\alpha)=\alpha$.

Proof: Suppose $\alpha$ is fuzzy gp*-closed subset in $Y$. Now by Theorem 6.2 (b) $\alpha \leq$ fuzzy $g p^{*}-\operatorname{cl}(\alpha)$. Also $\alpha \leq \alpha \&$ given that $\alpha$ is fuzzy gp*-closed set in $Y$, so by Theorem 6.2(c) fuzzy $g p^{*}$ $c l(\alpha) \leq \alpha$. Therefore we have fuzzy $g p^{*}-\operatorname{cl}(\alpha)=\alpha$.

Theorem 6.4 Suppose $\alpha \& \mu$ are fuzzy subsets in fuzzy topological space $(Y, \tau)$. Then we have fuzzy $\mathrm{gp}^{*}$-cl $(\alpha \wedge$ $\mu) \leq$ fuzzy $g p^{*}-\operatorname{cl}(\alpha) \wedge f u z z y g p^{*}-\operatorname{cl}(\mu)$.

Proof: Suppose $\alpha \& \mu$ are fuzzy subsets in $Y$. Then clearly $\alpha$ $\wedge \mu \leq \alpha$ and $\alpha \wedge \mu \leq \mu$. Now by Theorem 6.2 (d) fuzzy $g p^{*_{-}}$ $c l(\alpha \wedge \mu) \leq f u z z y g p^{*}-c l(\alpha)$ and fuzzy $g p^{*}$-cl $(\alpha \wedge \mu) \leq f u z z y$ $g p^{*}-c l(\mu)$. Implying that

fuzzy $g p^{*_{-}} \operatorname{cl}(\alpha \wedge \mu) \leq f u z z y g p^{*}-\operatorname{cl}(\alpha) \wedge f u z z y g p^{*}-c l(\mu)$.

Theorem 6.5 Suppose $(Y, \tau)$ is a fuzzy topological space and $\alpha$ is a fuzzy set in $Y$, then fuzzy $\operatorname{gp}^{*}-\operatorname{cl}(\alpha) \leq \operatorname{cl}(\alpha)$.

Proof: Suppose $\alpha$ is a fuzzy subset in $Y$. Now, we know that $\operatorname{cl}(\alpha)=\wedge\{\pi: \alpha \leq \pi, \pi$ is fuzzy closed $\}$. So if $\{\alpha \leq \pi: \pi$ is fuzzy closed set in $Y\} \Rightarrow\{\alpha \leq \pi: \pi$ is fuzzy gp*-closed set in $Y\}$, as every fuzzy closed set is fuzzy gp*-closed. So by Theorem 6.2(c) fuzzy $g p^{*}-\operatorname{cl}(\alpha) \leq \pi$. Therefore fuzzy $g p^{*}-\operatorname{cl}(\alpha) \leq \wedge\{\pi$ : $\alpha \leq \pi, \pi$ is fuzzy closed $\}=\operatorname{cl}(\alpha)$ I.e. fuzzy $g p^{*}-\operatorname{cl}(\alpha) \leq \operatorname{cl}(\alpha)$.

\section{REFRENCES}

1. L.A.Zadeh, Fuzzy Sets, information and control (1965) pp. 338-353.

2. C.L Chang, On Fuzzy Topological spaces, journal of mathematical analysis and applications, (1968) pp. 182-190.

3. R.H.Warren, continuity of mappings of fuzzy topological spaces, notices Amer. Math. Soc. 21 (1974), A-451.

4. G.P.Siddapur and S.S Benchalli, Fuzzy $g *$ PreContinuous Maps in Fuzzy Topological Spaces, International Journal of Computer Application. Volume 16- No. 2, (2011)

5. H.R. Moradi and A Kamali, On fuzzy strongly $g *_{\text {- }}$ closed sets and $g * *$-closed sets, International Journal Of Advances In Applied Mathematics and Mechanics (2015) pp. 13-17.

6. T. Fukutake, R.K.Saraf, M.caldas and S.Mishra, Mappings via Fgp-closed sets, Bull. Of Fukuoka, (2003) pp. 11-20.

7. Firdose Habib and Khaja Moinuddin, Fuzzy $g p^{*}$ closed sets in fuzzy topological spaces, South East Asian J. of Mathematics and Mathematical Sciences Vol. 16, No. 2 (2020), pp. 151-160. 